\title{
What Drives a Successful Adoption of E-Learning Modules for Sustainable Management? An Empirical Investigation of Influencing Factors and Challenges
}

\author{
Jan Moritz Anke \\ University of Goettingen \\ janke@uni-goettingen.de
}

\author{
Sebastian Hobert \\ University of Goettingen \\ shobert@uni-goettingen.de
}

\author{
Matthias Schumann \\ University of Goettingen \\ mschuma1@uni-goettingen.de
}

\begin{abstract}
In order to have corporations contributing to the environmental and social challenges of the coming years, employees have to develop competences for sustainable management. These competences are necessary to reduce environmental impacts of production or to increase efficiency in terms of energy consumption. In this context, E-Learning modules can offer learner-centered scenarios in which employees can simulate the consequences of their decisions and thereby develop competences for sustainable management. However, such modules focusing on sustainable management have not been used widely. Therefore, the aim of this paper is to investigate the determinants of a successful adoption and the challenges facing this adoption process of E-Learning modules for sustainable management. To achieve this aim, we conducted a literature review and a qualitative interview study. Thereby, we identified eleven general and eight specific influencing factors as well as twelve related challenges that affect the adoption of ELearning modules for sustainable management.
\end{abstract}

\section{Introduction}

In light of global sustainable challenges such as overexploitation of natural resources [6], businesses can help address these problems by e.g. employing technologies that are more efficient and logistics systems to reduce their impact on the environment [12]. In this context, employees need to develop competences for sustainable management. These competences help them review all their processes and determine their impact on the business and its environment [12]. In this respect, E-Learning modules can offer opportunities for the competence development of sustainable management by creating virtual learning scenarios that create authentic decision-making situations. In these situations, individuals can train their skills, which is the basis to gain competences [25]. One of the domain experts who has participated in our study summarizes this potential as follows:

"With the help of E-Learning modules, sustainable situations can be simulated that are difficult to reproduce in traditional classrooms." (E-Learning expert 1)

Nevertheless, E-Learning modules focusing on the competence development in the domain of sustainable management have not been used widely [1]. Thus, we need to analyze how to promote the adoption of these modules. Due to missing of influencing factors targeting the specific domain of sustainable management, we conduct a two-step procedure: First, we want to identify influencing factors in the literature that are related to E-Learning modules in general. Second, we combine these findings with specific influencing factors that we derive from an interview study. In doing so, we strive to promote the adoption of E-Learning modules in the specific domain of sustainable management. Hence, we ask the following research question:

RQ1: What is the state of the art regarding factors that influence the adoption of E-Learning modules?

To answer this research question, we conduct a literature review on influencing factors for E-Learning modules. Afterward, we employ a semi-structured interview study to focus on the specific influencing factors for adopting E-Learning modules for sustainable management to supplement the generic influencing factors. Therefore, we ask the second research question:

RQ2: What are additional factors influencing the adoption of E-Learning modules for sustainable management? 
Despite the massive opportunities provided by ELearning modules for sustainable management, there exist some challenges influencing the adoption of these modules by learners. Thus, the third research question is:

RQ3: What are specific challenges that determine the successful implementation of E-Learning modules for sustainable management?

In order to answer these research questions, the rest of the paper proceeds as follows: In the next section, we outline the basics on E-Learning modules for sustainable management as well as the theoretical framework used in this study. Afterward, we present the methodology and both general and specific influencing factors adopting of E-Learning modules for sustainable management in the findings, respectively. Thereafter, we discuss our findings. Then, we present limitations and future research. Finally, we summarize our findings in the conclusion section.

\section{Basics and theoretical framework}

In the following, we present the basics of ELearning modules for sustainable management. Afterward, we provide theoretical background information on the Technology-OrganizationEnvironment framework.

\subsection{E-Learning for sustainable management}

E-Learning is a type of learning that "can encompass all electronically supported learning and teaching" [15]. It can cover a range of tools such as computer-based and web-based courses [8]. ELearning modules are referred to as didactically linked learning objects, which are organized in the form of modular learning contents that convey a concrete topic [10]. Multimedia content such as interactive simulations can facilitate learning in real-world situations [4]. These authentic situations, in which individuals can apply their skills, are the basis for the competence development [32].

The aim of sustainable management is to control the ecological, social and economic effects of business activities [17] to serve the sustainable development not only of an organization but ultimately of a society [9]. To be able to act in sustainable ways in living and working environments, competences are needed [7]. In general, competences refer to the application of knowledge in concrete situations [29]. Competences for sustainable management are necessary to understand the complex construct of sustainability [9] and are therefore one of the desired outcomes of education for sustainable development [16]. Competences for sustainable management are further defined as "a complex ability to act adequately in business contexts, in particular, to be able to take into account the medium- and long-term economic, ecological and social - intra-company as well as external - consequences of management decisions" [27].

In prior researches, E-Learning modules were primarily considered or designed to just provide flexible access to sustainability-related information, such as an online lecture. Since most of these modules do not reflect authentic contexts essential to develop competences [30], these modules often not have the ability to create an effective learning environment. Moreover, the adoption of these E-Learning modules for competence development in sustainability context has not been targeted yet. Previous studies deal with the adoption of E-Learning modules without sustainability as the learning content. Therefore, an integrative view of the determinants of the adoption of E-Learning modules with sustainability as a learning content is missing [1].

\subsection{Theoretical framework}

According to the Technology-OrganizationEnvironment framework of DePietro et al. [14], the three dimensions, technology, organization and environment, are the three major determining factors, which may positively or negatively influence the decision to adopt a new technology. In prior research, numerous studies showed that this framework is suitable for analyzing the adoption of E-Learning modules [3]. Figure 1 depicts the TechnologyOrganization-Environment framework.

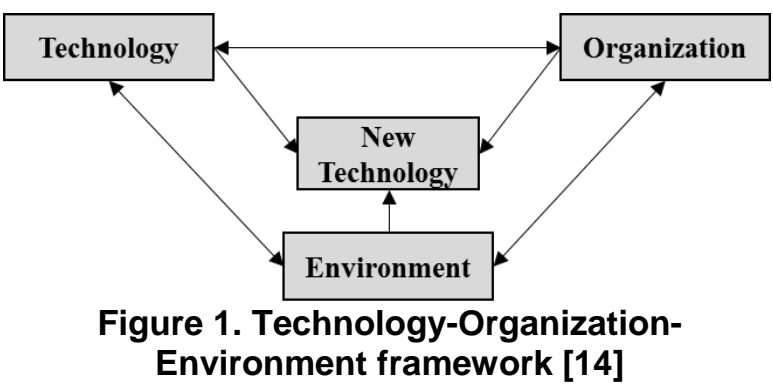

\section{General influencing factors for the adoption of E-Learning modules}

In order to provide comprehensive findings for influencing factors of adopting E-Learning modules for sustainable management, we first focus on general 
factors influencing the adoption of E-Learning modules. Therefore, we present the methodology and findings of a literature review in the following subsections.

\subsection{Methodology}

In order to answer RQ1, we conducted a literature review [31]. As the Technology-OrganizationEnvironment framework is suitable to analyze the adoption process of technologies such as E-Learning modules (see section 2.2), we searched on Google Scholar using the two terms: "E-Learning" and "Technology-Organization-Environment". We reviewed the titles and abstracts of the 380 results. At the next step, we excluded 374 articles, since their research focuses are different from ours. Following this, we analyzed six articles that are relevant and classified them independently according to the Technology-Organization-Environment framework. Thereby, we successively summarized similar factors and extended the three categories of this framework.

\subsection{Findings}

Based on the literature review, we identified eleven factors that influence the adoption of E-Learning modules. We classified them according to the Technology-Organization-Environment framework of DePietro et al. [14]. Table 1 summarizes the classification of the identified literature.

Table 1. Classification of the identified literature

\begin{tabular}{|c|c|c|c|c|c|c|c|c|}
\hline \multirow{2}{*}{\multicolumn{2}{|c|}{ Influencing factor }} & \multicolumn{6}{|c|}{ Articles } & \multirow{2}{*}{$\Sigma$} \\
\hline & & [2] & [3] & [22] & [23] & [24] & [26] & \\
\hline \multirow{3}{*}{ 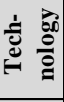 } & IT infrastructure (T1) & $\bullet$ & $\bullet$ & $\bullet$ & & $\bullet$ & $\bullet$ & 5 \\
\hline & Perceived ease of use (T2) & $\bullet$ & $\bullet$ & $\bullet$ & $\bullet$ & & & 4 \\
\hline & Technological compatibility (T3) & & & & $\bullet$ & & & 1 \\
\hline \multirow{5}{*}{ 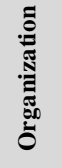 } & Expected benefits $(\mathrm{O} 1)$ & $\bullet$ & & $\bullet$ & $\bullet$ & & $\bullet$ & 4 \\
\hline & Size of the institution $(\mathrm{O} 2)$ & & & & $\bullet$ & & & 1 \\
\hline & Organizational compatibility (O3) & $\bullet$ & $\bullet$ & & & $\bullet$ & $\bullet$ & 4 \\
\hline & Top management support (O4) & & & & $\bullet$ & & & 1 \\
\hline & IT knowledge (O5) & & & & $\bullet$ & $\bullet$ & & 2 \\
\hline \multirow{3}{*}{ 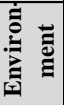 } & Educational partners (E1) & $\bullet$ & $\bullet$ & & & $\bullet$ & $\bullet$ & 4 \\
\hline & Competetitive pressure (E2) & $\bullet$ & $\bullet$ & $\bullet$ & $\bullet$ & $\bullet$ & $\bullet$ & 6 \\
\hline & Regulatory policy (E3) & & & & $\bullet$ & & & 1 \\
\hline
\end{tabular}

In the following, we present the technological, organizational and environmental influencing factors, respectively.

3.2.1. Technological influencing factors. In the first category, we identified three general technological influencing factors for the adoption of E-Learning modules. These factors include technologies that have already been exploited and the ones that have not been in use but are available on the market [5]. Five articles named the IT infrastructure (T1) as an influencing factor. IT infrastructure includes a fast Internet connection, new computers and a high-quality computer network [26]. Moreover, four articles indicated that the perceived ease of use (T2) of the ELearning modules is an influencing factor. Perceived ease of use refers to the extent to which potential users expect the E-Learning module to be free of effort [13]. Therefore, users of these modules should comfortably use it. This includes advantages such as shortening the loading time for learning materials [2] and reducing the complexity of the system [23]. The last technological factor, mentioned in one article, is the technological compatibility (T3). This is defined as the degree to which E-Learning modules are consistent with the adopter's current technological requirements [23].

3.2.2. Organizational influencing factors. We identified five organizational factors in the literature that influence the adoption of E-Learning modules. These factors include the structure and characteristics of an organization [5]. The first influencing factor, noted in four articles, targets the expected benefits (O1) of E-Learning modules. The expected benefits refer to the extent that the advantages of new ELearning modules outperform that of traditional learning approaches [26]. Examples include increased user satisfaction and improved academic performance [22]. When more expected benefits of E-Learning modules are realized, it is more likely that institutions allocate more financial, managerial and technological resources to implement these modules [23]. The size of the institution (O2) is another facilitator for the adoption of technological innovations that was suggested in one article. In this context, large institutions have a greater risk-taking endurance. Such institutions can allocate more financial resources on ELearning modules compared to small institutions [23]. Four experts indicated that the organizational compatibility (O3) is an influencing factor. This refers to the level to which the E-Learning modules are compatible with the institutions' objectives or operations [24]. Another factor for the adoption of ELearning modules is the top management support (04). According to [23], the top management support contributes to the adoption of E-Learning modules by creating an affirmative environment for E-Learning. For example, the top management can provide financial and human resources, reduce barriers and increase willingness to change. The last influencing factor in this category, mentioned in two articles, is the IT knowledge (O5). In this context, it is important that 
learners are technologically prepared to use the ELearning modules, and that human resources and technological infrastructure are ready to support the adoption [24].

3.2.3. Environmental influencing factors. In the third category, we were able to derive three environmental factors influencing the adoption of E-Learning modules. These factors describe surrounding conditions such as regulatory guidelines and laws [14]. Four articles mentioned educational partners (E1) as one of the environmental factors. These educational partners such as governments or teachers have good knowledge about the benefits of E-Learning modules and how to adopt them [3]. They can help an institution to successfully adopt these modules. Additionally, all articles noted that competitive pressure (E2) is an important environmental factor for the adoption of ELearning modules. Competitive pressure erupts in an institution when it is losing competitive advantages against other institutions. The implementation of ELearning modules can open new opportunities in the field of teaching and provide a good reputation for the institution [3]. In this context, an institution that implements E-Learning modules can gain a competitive advantage. The last influencing factor, noted by one article, is the regulatory policy (E3). For example, governments can introduce regulations that force institutions to adopt and implement certain technology standards. Consequently, E-Learning modules need to meet these legal rules [23, 33].

\section{Specific influencing factors for the adoption of E-Learning modules for sustainable management}

Since we did not identify any specific influencing factors for adopting E-Learning modules for sustainable management during the literature review, we used an explorative approach to derive specific influencing factors. We conducted an interview study. In the following, we introduce the underlying methodology and findings.

\subsection{Methodology}

To complement the findings from section 3 and to identify influencing factors (RQ2) as well as related challenges (RQ3) for the adoption of E-Learning modules for sustainable management, we conducted a qualitative and exploratory interview study with 23 experts in the E-Learning and sustainability domains. The interviewees have at least three years of work experience in their respective domain. As displayed in
Figure 2, we divided our methodological approach into three steps.

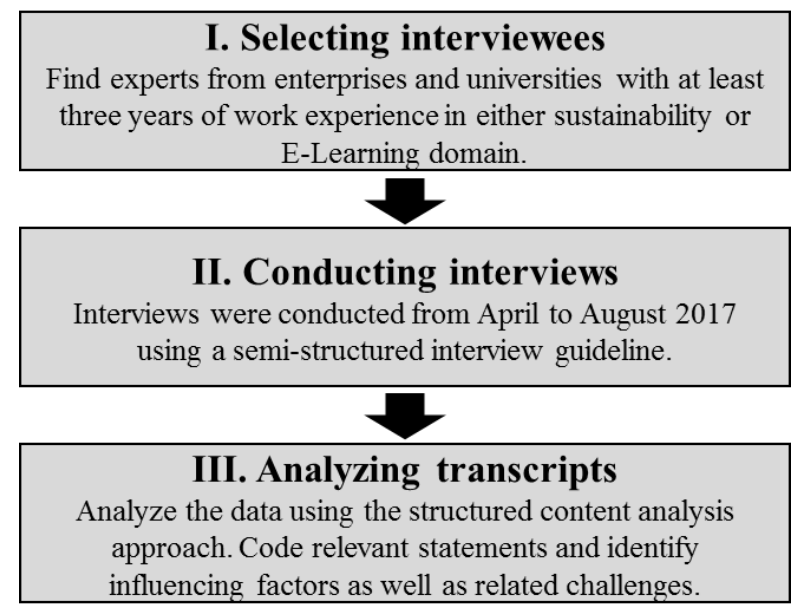

Figure 2. Research design

First, to ensure a comprehensive view on influencing factors and related challenges of adopting E-Learning modules for sustainable management, we selected potential experts from enterprises and universities in Germany. This heterogeneous view is also the reason of selecting experts from sustainability and E-Learning domains such as Corporate Social Responsibility (CSR) consultants or E-Learning managers. We sent invitation emails for conducting an interview to potential experts. In total, 10 experts from the sustainability domain (SE1-SE10) and 13 ELearning experts (EE1-EE13) accepted our interview invitation, resulting in an acceptance rate of around $21 \%$. Table 2 shows the summarized characteristics of the sample.

Table 2. Sample characteristics

\begin{tabular}{|l|l|l|}
\hline Expert & Position of expert & Industry \\
\hline SE1 & CSR Consultant & Education \\
\hline SE2 & CSR Consultant & Education \\
\hline SE3 & Professor for CSR & Education \\
\hline SE4 & CSR Consultant & Retail \\
\hline SE5 & CSR Manager & Consulting \\
\hline SE6 & CSR Consultant & Mining \\
\hline SE7 & Environmental Scientist & Education \\
\hline SE8 & CSR Consultant & Education \\
\hline SE9 & CSR Consultant & Automotive \\
\hline SE10 & CSR Director & Retail \\
\hline EE1 & E-Learning Developer & Education \\
\hline EE2 & E-Learning Developer & Education \\
\hline EE3 & E-Learning Consultant & Education \\
\hline EE4 & E-Learning Manager & Automation \\
\hline EE5 & E-Learning Manager & Banking \\
\hline EE6 & E-Learning Manager & Education \\
\hline
\end{tabular}




\begin{tabular}{|l|l|l|}
\hline EE7 & E-Learning Consultant & Healthcare \\
\hline EE8 & E-Learning Manager & Education \\
\hline EE9 & E-Learning Manager & Education \\
\hline EE10 & E-Learning Consultant & Security \\
\hline EE11 & E-Learning Manager & Education \\
\hline EE12 & E-Learning Manager & Consulting \\
\hline EE13 & E-Learning Manager & Education \\
\hline
\end{tabular}

Second, we conducted interviews via phone or face-to-face from April to August 2017. We used a semi-structured interview guideline to conduct the interviews, to leave the interviewees enough freedom to express their own ideas [21]. In the last interviews, we did not reveal any new insights. At this point, we decided not to conduct further interviews, since the theoretical saturation has been reached [18]. In order to allow an in-depth analysis of the interviews, we asked all domain experts if we were allowed to record the interviews on tape and transcribe them later. All interviews were recorded with an average of 28 minutes per interview. We conducted the interviews in German, as all experts were German. In order to publish the results of this study, we translated the transcripts from German into English following the constant contextual comparison [28].

Third, we analyzed our data by using the structured content analysis approach [19]. Therefore, we coded relevant statements in the transcripts and constantly discussed them to identify the core topic of our study: influencing factors and related challenges of adopting E-Learning modules for sustainable management

\subsection{Findings}

Based on the interview study, we identified eight specific influencing factors that can influence the adoption of E-Learning modules for sustainable management. Again, we classified them according to the Technology-Organization-Environment framework. In the following, we present specific technological, organizational and environmental influencing factors and twelve related challenges facing the adoption of ELearning modules for sustainable management.

4.2.1. Technological influencing factors. In the first category, we identified one technological influencing factor and two related challenges. The experts mentioned the mobile accessibility (T4) as a technological influencing factor. Since learning about sustainability often takes place on site, like as part of an excursion to a forest, it is important that the ELearning modules are accessible from anywhere with all kinds of electronic devices (TC4.1). Our interviewees stated that especially in the field, the mobile data coverage could not always be guaranteed. Moreover, the internet connection, for example, in the woods is not always good enough to allow quick download of big data packages such as videos or simulations (TC4.2). Table 3 depicts the technological influencing factor, quotations and related challenges.

Table 3. Technological influencing factor T4: Mobile accessibility

\section{Quotations}

"Make sure everyone has access [to the E-Learning modules] from everywhere." (EE3)

"Especially new generations say "mobile only"." (EE3)

Challenges

TC4.1: Difficulties with the accessibility of mobile data on excursions

TC4.2: Difficulties with download of large data packages

4.2.2. Organizational influencing factors. In the category of organizational influencing factors, we were able to derive three such factors and six challenges. The first organizational factor influencing the successful adoption of E-Learning modules for sustainable management is the integration into the curriculum (O6). The curriculum should provide users with enough time to become familiar with and complete E-Learning modules for sustainable management (OC6.1). If no additional time is provided for these modules or if it is seen as an extra work, the acceptance and thereby the adoption of these modules is limited. Moreover, the use of these modules can only be successful if the users are encouraged to use the modules (OC6.2). For example, providing gamification elements can improve users' involvement and learning about sustainability. Table 4 exemplifies quotations describing this organizational influencing factor and summarizes the related challenges.

Table 4. Organizational influencing factor (1/3)

\begin{tabular}{|l|}
\hline \multicolumn{1}{|c|}{ O6: Integration into the curriculum } \\
\hline Quotations \\
"In order to work on the E-Learning modules, there \\
must also be some form of incentive in place." \\
(EE8) \\
\hline "Even if it is firmly anchored in the curriculum, \\
there should be time for taking part in the E- \\
Learning modules for sustainable management. I \\
think it is also very important that you don't end up \\
with extra work." (EE10) \\
\hline Challenges \\
OC6.1: Provide extra time \\
OC6.2: Excite users \\
\hline
\end{tabular}


In addition to the curriculum, the integration into the sustainability concept (O7) of an institution influences the successful adoption of E-Learning modules for sustainable management (see Table 5). The content of the E-Learning modules has to match the sustainability concept of an institution (OC7.1). Furthermore, a contact person, who has competences in E-Learning as well as in sustainability and is available for answering queries, is necessary (OC7.2). This person should help users with technical and contentrelated problems and further encourage the users of the modules to develop their competences in sustainable management.

Table 5. Organizational influencing factor $(2 / 3)$ O7: Integration into the sustainability concept Quotations

"Sustainability must, therefore, be an integral part of the organization's philosophy, and this makes it possible to act very successfully." (SE4)

"You can see that the economic and also the ecological necessity that things have to change, but integration is difficult." (SE5)

"There should always be a permanent contact person who is also responsible for problems of all kinds, whether this is for IT or a kind of

moderator." (EE10)

Challenges

OC7.1: Content has to match sustainability concept

OC7.2: Contact person with knowledge in

sustainability and E-Learning

Moreover, the willingness to adopt E-Learning modules for sustainable management is influenced by the learning culture (O8). Since competences are described as learnable but not teachable [32], users can only develop competences for sustainable management by taking a more active role in the learning process (OC8.1). Especially for questions regarding sustainability, an active and discussion-based approach is necessary, since these questions do not always have a clearly right or wrong answer. As an example, it has not been clarified yet how sustainable electric cars are. For example, the life cycle assessment depends on whether the electricity comes from renewable energy sources [11]. Therefore, the adoption of these modules depends on the users' willingness to discuss and actively learn. However, according to the interview partners, enhancement of the willingness to discuss these topics with the help of electronic devices, for example, in an online forum, is a challenge (OC8.2). The experts believe that the lack of human interaction in E-Learning modules is a cause. Table 6 exemplifies a quotation and related challenges.
Table 6. Organizational influencing factor (3/3)

\begin{tabular}{|l|}
\hline \multicolumn{1}{|c|}{ O8: Learning culture } \\
\hline Quotations \\
"Often we are still used to [listen to a presentation]. \\
Now in E-Learning modules, a lot has changed: the \\
learner is in a much more active role and therefore \\
must show self-discipline. This fact must first \\
become conscious and clear and the user must be \\
ready for it and also be well guided." (EE3) \\
"Sustainability topics are often discursive topics \\
and this discursivity or willingness to discuss may \\
be less in E-Learning." (SE2) \\
\hline Challenges \\
OC8.1: Active learning \\
OC8.2: Encouraging the willingness to discuss \\
\hline
\end{tabular}

4.2.3. Environmental influencing factors. Based on our empirical study, we identified two environmental influencing factors and four challenges. The first environmental influencing factor, mentioned by the experts, is the importance of sustainability (E4). Only if sustainability has a certain value and relevance in society in the respective environment, the adoption of the modules can be successful (EC4.1). However, our interviewed experts recognized that in some countries sustainable management is undervalued (EC4.2). Especially if governments and other stakeholders do not support sustainable management, the adoption of these modules is less likely to be successful. One expert notes that for many institutions in Germany, it is mandatory to report on sustainability matters. Thus, employers and other members of these institutions must be educated in this matter. E-Learning education for sustainability is important in this country and their users are more likely to adopt E-Learning modules for sustainable management. Moreover, it is a challenge to illustrate the importance of sustainability aspects in a changing society (EC4.3). One interviewee indicates that presenting the business aspects of sustainability is more convincing to potential users rather than presenting the E-Learning modules as an eco-program. Moreover, it should be explained that sustainable development can ensure the survival of the institution and is an integral part of the sustainability concept. Table 7 shows this environmental influencing factor.

Table 7. Environmental influencing factor (1/2) E4: Importance of sustainability

\section{Quotations}

"[...] you naturally have very different assessments depending on which culture and country you work in because the regulations are different. And it means something completely different to make a 
decision about sustainability in China or in

Germany." (SE9)

"[It should be stated] that the E-Learning module is not only about environmental protection, but also about the continued existence of the institution." (SE6)

Challenges

EC4.1: Lack of importance of sustainability

EC4.2: Openness for sustainable management varies regionally

EC4.3: Illustrating the significance of sustainable management

In addition to the importance of sustainability, the experts identified data protection (E5) as an environmental influencing factor (see Table 8). Especially because personal data can be stored during the assessment of competence for sustainable management in the E-Learning modules, data protection is needed. For this reason, it is important to follow the data protection guidelines. For example, the EU has a very restrictive legal basis that restricts the storage and transfer of personal data [20]. One aspect of data protection is data security. Experts agreed that confidential data must be protected from unauthorized access or external destruction (EC5.1). In addition, data collected should be protected from manipulation.

Table 8. Environmental influencing factor (2/2) E5: Data protection

Quotations

"Data protection is relevant, especially if you want to store and evaluate additional data such as processing time and click paths. Then you have to keep in mind that the users agree your terms of condition and that the whole thing is in accordance with the data protection officer or the data protection rules." (EE1)

"Security must be guaranteed when storing personal data. In this way, no strangers can check on others' learning progress." (EE1)

\section{Challenge}

EC5.1: Provide adequate data security protection

\section{Discussion}

Since the identified eleven general influencing factors from the literature review are insufficient to provide a comprehensive overview of all factors that are likely to facilitate or hinder the adoption of ELearning modules for sustainable management, we derive six specific influencing factors and twelve related challenges targeting this adoption process.
Accordingly, all factors and challenges need to be considered when introducing these E-Learning modules.

When analyzing the influencing factors of the adoption of E-Learning modules for sustainable management, most experts state that the technological influencing factors will not be critical in the future because of the rapid expansion of the broadband for mobile data. Moreover, most organizations have had already a good infrastructure and most of the ELearning modules are easy to integrate into the technological system. Hence, this will not be a challenge in the future.

However, organizational influencing factors are more important: In particular, the experts rate the learning culture $(\mathrm{O} 8)$ as critical, because in most of the organizations, the active learning culture is still underdeveloped and the willingness to discuss sustainable problems is limited. Nevertheless, the remaining organizational influencing factors are less critical as they can be solved if planned carefully in advance. Therefore, it is necessary to proactively involve the top management during the implementation process and excite potential users to take part in the modules. The potential users can be motivated by communicating benefits and expectations of these ELearning modules and give them enough time to participate in these modules. This is important to prevent resistance and face the related challenges. Moreover, the integration into the sustainability concept will be accepted in the future, as there is a shift towards more sustainable management in many organizations.

Finally, the environmental influencing factors must be taken into account. In particular, the experts indicate that the importance of sustainability (E4) is critical in certain countries. Therefore, it is becoming more important to communicate the benefits of these modules. In contrast, other challenges such as providing adequate data security protection (E5) will be solvable if it is considered in advance to potential use.

Figure 3 summarizes the general and specific identified influencing factors of adopting E-Learning modules for sustainable management as well as their rating.

Nevertheless, it should be noted that the use of these modules can only be successful if the content and the layout of the E-Learning modules are carefully designed. Some experts state that the instruction and the curriculum should not be directly pulled from books but make use of all advantages of interactive learning like simulations. It can provide a better illustration of the complexity of sustainability and therefore, users are able to have a better understanding 
of the topic. Furthermore, the authenticity of the ELearning modules is important for the successful adoption of E-Learning modules for sustainable management.

\section{Limitations and future research}

Although this study focuses on E-Learning modules for sustainable management, most of the influencing factors and their related challenges can easily be extended to the entire field of E-Learning modules. As with any literature review and interview study, we recognized that our research entails some limitations that the reader should keep in mind while interpreting the results: First, we acknowledge that it is possible that we did not include all existing articles to ELearning and the Technology-OrganizationEnvironment framework in our literature review. The review is limited to Google Scholar and the used keywords. Second, our findings in the interview study are highly dependent on the selection of the interviewees. We selected experts from universities and different industry sectors such as healthcare and automotive. However, we only interviewed German experts and that can lead to a limited generalizability. As such, future research should examine more than one country to provide a comparison and validation of the results. Third, the interpretation of qualitative data is always subjective. To address this problem, we tried to minimize subjective influences by applying the structured content analysis approach and coded the statements with three persons.

\section{Conclusion}

The aim of this study is to promote the adoption of E-Learning modules for sustainable management. Hence, we wanted to identify factors that are likely to help or hinder the adoption of these modules. Therefore, we conducted a literature review to derive general influencing factors of the adoption of ELearning modules and an interview study among 23 experts from the sustainability and E-Learning domains to supplement specific influencing factors targeting the adoption of E-Learning modules for sustainable management. Based on these studies, we identified eleven general (RQ1), six specific influencing factors (RQ2) and twelve related challenges (RQ3) in the three context categories of the Technology-Organization-Environment framework. This study bridges the literature gap by providing influencing determinants and related challenges of the adoption of E-Learning modules, especially for sustainable management. Moreover, scholars can use these insights to analyze the influential factors and related challenges of E-Learning modules in other domains for a more generalized view. Furthermore, our results can constitute a basis for future studies of actual

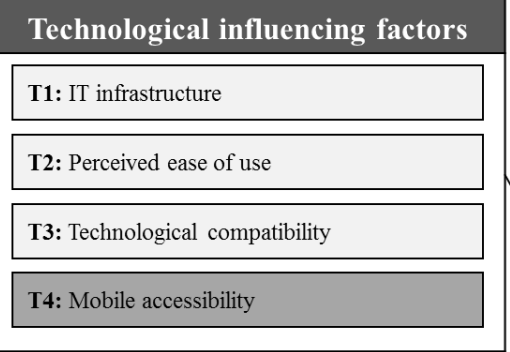

$\square$ General influencing factor
$\square$ Specific influncing factor
$*$ critical
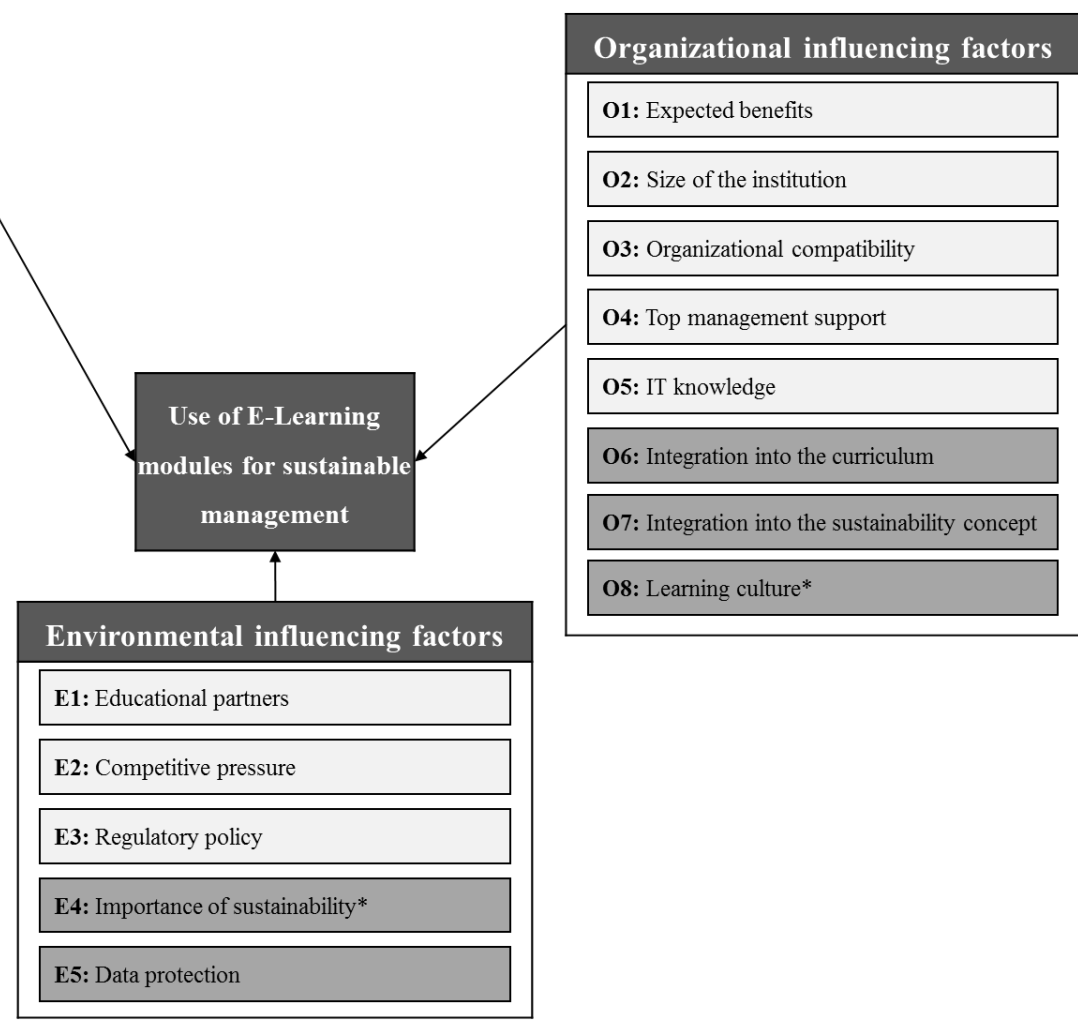

Organizational influencing factors

Figure 3. General and specific influencing factors 
system implications. Thereby, our results may help improve the adoption of E-Learning modules for sustainable management, since it exemplifies and explains potential challenges.

\section{References}

[1] Anke, J.M. and M. Schumann, "E-learning modules supporting education for sustainable development - state of the art and future research directions", in EDULEARN17 Proceedings, L. Gómez Chova, A. López Martínez, and I. Candel Torres, Editors, International Conference on Education and New Learning Technologies, Barcelona, Spain. 2017. IATED.

[2] Ansong, E., S.L. Boateng, and R. Boateng, "Determinants of E-Learning Adoption in Universities", Journal of Educational Technology Systems, 46(1), 2017, pp. 30-60.

[3] Ansong, E., S.L. Boateng, R. Boateng, and J. Effah, "Determinants of E-Learning Adoption in Universities: Evidence from a Developing Country", in 49th Hawaii International Conference on System Sciences (HICSS), T.X. Bui and R.H. Sprague, JR., Editors, Koloa, HI, USA. 2016.

[4] Azeiteiro, U.M., P. Bacelar-Nicolau, F.J. Caetano, and S. Caeiro, "Education for sustainable development through elearning in higher education: Experiences from Portugal", Journal of Cleaner Production, 106, 2014, pp. 308-319.

[5] Baker, J., "The Technology-Organization-Environment Framework", in Information Systems Theory: Explaining and Predicting Our Digital Society, K.Y. Dwivedi, R.M. Wade, and L.S. Schneberger, Editors. 2012: New York.

[6] Barbier, E.B., "Natural capital and labor allocation: Mangrove-dependent households in Thailand", The Journal of Environment \& Development, 16(4), 2007, pp. 398-431.

[7] Barth, M., J. Godemann, M. Rieckmann, and U. Stoltenberg, "Developing key competencies for sustainable development in higher education", International Journal of Sustainability in Higher Education, 8(4), 2007, pp. 416-430.

[8] Bitzer, P., K. Lehmann, and J.M. Leimeister, "A Literature Review on Indicators for the Measurement of Technology Mediated Learning Productivity: 2000 to 2011", in AMCIS 2012 Proceedings, Seattle; Washington; USA, 2012. 2012: Seattle, Washington.

[9] Cebrián, G. and M. Junyent, "Competencies in Education for Sustainable Development: Exploring the Student Teachers' Views", Sustainability, 7(3), 2015, pp. 2768-2786.

[10] Cohen, E. and M. Nycz, "Learning objects and elearning: An informing science perspective", Interdisciplinary Journal of E-Learning and Learning Objects, 2(1), 2006, pp. 23-34.
[11] Dang, S., A. Odonde, T. Mirza, C. Dissanayake, and R. Burns, "Sustainable energy management: An analysis report of the impacts of electric vehicles", in 14th International Conference on Environment and Electrical Engineering (EEEIC), IEEE, Editor. 2014. IEEE: Piscataway, NJ.

[12] Daub, C-H and R. Ergenzinger, "Enabling sustainable management through a new multi-disciplinary concept of customer satisfaction", European Journal of Marketing, 39(9/10), 2005, pp. 998-1012.

[13] Davis, F.D., "Perceived Usefulness, Perceived Ease of Use, and User Acceptance of Information Technology", MIS Quarterly, 13(3), 1989, pp. 319-340.

[14] DePietro, R., E. Wiarda, and M. Fleischer, "The context for change: Organization, technology and environment", in The process of technological innovation, L.G. Tornatzky and M. Fleischer, Editors. 1990: Lexington.

[15] Diamond, S. and B. Irwin, "Using e-learning for student sustainability literacy: Framework and review", International Journal of Sustainability in Higher Education, 14(4), 2013, pp. 338-348.

[16] Dlouhá, J. and S. Burandt, "Design and evaluation of learning processes in an international sustainability oriented study programme. In search of a new educational quality and assessment method", Journal of Cleaner Production, 106, 2015, pp. 247-258.

[17] Elkington, J., Cannibals with Forks: Triple Bottom Line of 21st Century Business, Oxford, 1997.

[18] Glaser, B.G., Theoretical Sensitivity, San Francisco, 1978.

[19] Mayring, P., Qualitative content analysis: theoretical foundation, basic procedures and software solution, Klagenfurt, 2014.

[20] Mostert, M., A.L. Bredenoord, M.C.I.H. Biesaart, and J.J.M. van Delden, "Big Data in medical research and EU data protection law: challenges to the consent or anonymise approach", European journal of human genetics: EJHG, 24(7), 2016, pp. 956-960.

[21] Myers, M.D., Qualitative research in business \& management, SAGE, London, 2013.

[22] Namisiko, P., C. Munialo, and S. Nyongsea, "Towards an Optimization Framework for E-Learning in Developing Countries: A Case of Private Universities in Kenya", Journal of Computer Science and Information Technology, 2(2), 2014, pp. 2334-2374.

[23] Nyeko, J.S. and C. Ogenmungu, "Determinants of Electronic Learning Adoption in Higher Institutions of Learning in Uganda: A Learners' Perspective", Global Journal of Computer Science and Technology, 17(1), 2017, pp. 7-20. 
[24] Nyeko, S. and M. Moya, "Determinants of eLearning Adoption Among Instructors in Ugandan Public Universities", in 2017 IST-Africa Week Conference: 31 May-02 June 2017, Windhoek, Namibia, P. Cunningham and M. Cunningham, Editors. 2017. IEEE: Piscataway, NJ.

[25] Pretorius, R., M. Nicolau, J. Immelman, A. de Jager, M. Nöthling, and A. Du Plessis, "Virtual Learning Environments for Transformation to Sustainability: A Case Study from the "South"", in Integrating Sustainability Thinking in Science and Engineering Curricula, W. Leal Filho, U.M. Azeiteiro, S. Caeiro, and F. Alves, Editors. 2015. Springer International Publishing: Cham.

[26] Raouf, J.B., I.S. Naser, and B.K. Jassim, "Determinants of e-Learning implementation success in the Iraqi MoHE", Eng. \& Tech. Journal, 30(4), 2012, pp. 659-671.

[27] Seeber, S., J. Hartig, S. Dierkes, and M. Schumann, "Ko-NaMa - Simulation-based Measurement and Validation of a Competence Model for Sustainability Management", in Modeling and Measuring Competencies in Higher Education - Validation and Methodological Innovations (KoKoHs) Overview of the Research Projects, H.A. Pandt, O. ZlatkinTroitschanskaia, C. Lautenbach, M. Toepper, and D. Molerov, Editors. 2016: Berlin, Mainz.

[28] Suh, E.E., S. Kagan, and N. Strumpf, "Cultural competence in qualitative interview methods with Asian immigrants", Journal of transcultural nursing, 20(2), 2009, pp. 194-201.

[29] Sydow, J., S. Duschek, G. Möllering, and M. Rometsch, Kompetenzentwicklung in Netzwerken: eine typologische Studie, Wiesbaden, 2003.

[30] van Dam-Mieras, R., A. Lansu, M. Rieckmann, and G. Michelsen, "Development of an Interdisciplinary, Intercultural Master's Program on Sustainability: Learning from the Richness of Diversity", Innovative Higher Education, 32(5), 2008, pp. 251-264.

[31] Vom Brocke, J., A. Simons, B. Niehaves, B. Niehaves, K. Reimer, R. Plattfaut, and A. Cleven, "Reconstructing the Giant: On the Importance of Rigour in Documenting the Literature Search Process.", Proceedings of the European Conference on Information Systems (ECIS), 2009, pp. 22062217.

[32] Weinert, F.E., "Concept of Competence: A Conceptual Clarification", in Defining and Selecting Key Competencies, D.S. Rychen and L.H. Salganik, Editors. 2001: Seattle.

[33] Zhu, K., S. Xu, and J. Dedrick, "Assessing Drivers of EBusiness Value: Results of a Cross-Country Study", ICIS 2003 Proceedings, 16, 2003, pp. 181-193. 